\title{
Espiritualidade no enfrentamento do tratamento oncológico: revisão integrativa da literatura
}

\author{
Spirituality in coping with cancer treatment: an integrative literature review
}

\section{Espiritualidad en el enfrentamiento del tratamiento oncológico: revisión integradora de la literatu- ra}

\author{
Joyce Santos Martins ${ }^{1 *}$, Juliana Xavier Pinheiro da Cunha ${ }^{2}$, Chrisne Santana Biondo ${ }^{3}$, Lívia dos \\ Santos Mendes.
}

Como citar esse artigo. Martins, JS; da Cunha, JXP; Biondo, CS; Mendes, LS. Espiritualidade no enfrentamento do tratamento oncológico: revisão integrativa da literatura. Revista PróUniverSUS. 2021 Jan./Jun.; 12 (1): $116-124$

\section{Resumo}

Objetiva-se analisar as evidências científicas sobre o exercício da espiritualidade em pacientes com câncer, tendo em vista suas contribuições para o tratamento oncológico. Realizou-se uma revisão integrativa da literatura nas bases de dados MEDLINE, LILACS, BDENF e literatura cinzenta. Foram selecionados 15 artigos em português, inglês e espanhol, publicados entre os anos de 2010 e 2020, que abordaram o papel da espiritualidade durante o tratamento oncológico. Para discussão dos resultados estabeleceu-se três categorias: (1) a espiritualidade e o bem-estar físico e emocional, (2) a espiritualidade na construção da resiliência, e (3) a espiritualidade ressignificando a vida. Notou-se que o aumento de aspectos positivos relacionados à prática espiritual como otimismo, esperança, adoção de hábitos saudáveis e redução do estresse e da ansiedade refletem no bemestar e na qualidade de vida; o exercício espiritual oferece mecanismos para construção da resiliência, como resistência, força e superação das adversidades; e ajudam os pacientes a enxergar a vida sob uma perspectiva mais otimista. Poucos estudos abordam que não há benefícios relacionados à pratica da espiritualidade. Conclui-se, conforme a maioria das pesquisas, que a prática espiritual é relevante para o bem-estar, enfrentamento da doença e ressignificação da existência durante o tratamento oncológico.

Palavras-chave: Espiritualidade; Oncologia; Adaptação Psicológica.

\begin{abstract}
The objective is to analyze the scientific evidence on the exercise of spirituality in cancer patients, in view of their contributions to cancer treatment. An integrative literature review was carried out in MEDLINE, LILACS, BDENF and grey literature databases. It was selected fifteen articles in Portuguese, English and Spanish, published between 2010 and 2020, which addressed the role of spirituality during cancer treatment. For discussion of the results, three categories were established: (1) spirituality and physical and emotional well-being, (2) spirituality in building resilience, and (3) spirituality giving new meaning to life. It was noted that the increase in positive aspects related to spiritual practice such as optimism, hope, adoption of healthy habits and reduction of stress and anxiety are reflected in well-being and quality of life; spiritual exercise offers mechanisms for building resilience, such as resistance, strength and overcoming adversity; and help patients see life from a more optimistic perspective. Few studies address that there are no benefits related to the practice of spirituality. It is concluded, according to most research, that spiritual practice is relevant to well-being, coping with the disease and re-signifying existence during cancer treatment.
\end{abstract}

Keywords: Spirituality; Medical Oncology; Adaptation Psychological.

${ }^{1}$ Discente do curso de enfermagem, Universidade Federal da Bahia, Vitória da Conquista, Bahia, Brasil. E-mail: enf.joycemartins@gmail.com ORCID: https://orcid.org/0000-00033007-7060.

${ }^{2}$ Enfermeira, doutoranda em epidemiologia pra Universidade Federal do Rio Grande do Sul, professora assistente da Universidade Federal da Bahia - UFBA, Vitória da Conquista, Bahia, Brasil. E-mail: julianaxcunha@gmail.com. ORCID: https://orcid.org/0000-0002-3752-206X.

${ }^{3}$ Enfermeira, doutoranda em ciências da saúde pela Universidade Estadual do Sudoeste da Bahia-UESB, professora assistente da Universidade Federal da Bahia-UFBA, Vitória da Conquista, Bahia, Brasil. E-mail: tity_biondo_enf@hotmail.com. ORCID: https://orcid.org/0000-0002-0583-5491.

${ }^{4}$ Enfermeira, mestre em saúde coletiva pela Universidade Federal da Bahia-UFBA. Enfermeira assistente da Unidade de Terapia Intensiva Adulto do Hospital Geral de Vitória da Conquista, Bahia, Brasil. E-mail: liviaenfuesb@yahoo.com.br. ORCID: https://orcid.org/0000-0001-6053-8484.

* Email de correspondencia: enf.joycemartins@gmail.com 


\section{Resumen}

Tiene como objetivo analizar las evidencias científicas sobre el ejercicio de la espiritualidad en pacientes con cáncer, teniendo en vista sus contribuciones para el tratamiento oncológico. Se realizó una revisión integradora de la literatura en las bases de datos MEDLINE, LILACS, BDENF y literatura gris. Se seleccionaron 15 artículos en portugués, inglés y español, publicados entre 2010 y 2020, que abordaron el papel de la espiritualidad durante el tratamiento del cáncer. Para la discusión de los resultados, se estableció tres categorías: (1) espiritualidad y bienestar físico y emocional, (2) espiritualidad en la construcción de resiliencia, y (3) espiritualidad dando un nuevo significado a la vida. Se notó que el aumento de aspectos positivos relacionados con la práctica espiritual como optimismo, esperanza, adopción de hábitos saludables y reducción del estrés y de la ansiedad reflejan en el bienestar y en la calidad de vida; el ejercicio espiritual ofrece mecanismos para construcción de la resiliencia, como resistencia, fuerza y superación de las adversidades; y ayudan a los pacientes a ver la vida desde una perspectiva más optimista. Pocos estudios abordan que no existen beneficios relacionados con la práctica de la espiritualidad. Se concluye, de acuerdo con la mayoría de las investigaciones, que la práctica espiritual es relevante para el bienestar, enfrentamiento de la enfermedad y resignificación de la existencia durante el tratamiento oncológico.

Palabras clave: Espiritualidad; Oncología Médica; Adaptación Psicológica.

\section{Introdução}

A espiritualidade influencia o comportamento humano e tem relação com a busca de significado, paz e propósito. Ela não se limita a tipos de crenças ou práticas e pode contribuir com o bem-estar e enfrentamento das adversidades ${ }^{1}$. O ser humano é dotado desta esfera espiritual, sendo esta aflorada, ou não, de acordo com as vivências de cada indivíduo. Integrar-se com a própria espiritualidade pode ser uma experiência muito significativa, em especial no contexto saúde-doença, visto que amplifica o entendimento e compreensão das dificuldades, ressignificando o sofrimento ${ }^{2}$.

As práticas espirituais são comumente utilizadas por pacientes acometidos por câncer a fim de encontrar alívio para o sofrimento emocional e suporte para lidar com o estresse relacionado ao tratamento ${ }^{3}$. Este exercício espiritual, por sua vez, pode ser considerado um impulsionador da resiliência e do enfrentamento no cenário de adoecimento oncológico ${ }^{4}$.

O diagnóstico de câncer gera grandes mudanças na vida, na rotina e nas relações interpessoais, o que pode afetar as emoções pelas inseguranças consequentes do adoecimento por uma doença considerada grave e repleta de representações sociais negativas ${ }^{5}$. Neste contexto, a dor perpassa pelas dimensões física, social, emocional e espiritual ${ }^{6}$. Corroborando com estes resultados, constatou-se que pacientes oncológicos são mais propensos à sintomas depressivos, apresentam maior vulnerabilidade ao estresse e menor nível de esperança ${ }^{7}$.

O tratamento do câncer, é um dos principais fatores para o sofrimento físico e emocional relacionado à doença, por gerar medo e desconfortos devido aos efeitos colaterais de mal estar, à perda dos cabelos e à alteração da imagem corporal ${ }^{8}$. Os efeitos do tratamento interferem diretamente na qualidade de vida do paciente por aumentar o mal-estar, gerando indisposição ${ }^{9}$. Além disso, o processo de tratamento vai além do ambiente ambulatorial, resultando em desgastes físicos e psicológicos relacionados à trajetória dos pacientes desde o início dos sintomas até o tratamento, à locomoção, às mudanças de rotina e ao aumento do gasto financeiro ${ }^{10}$.

Diante de todo prejuízo físico, emocional e social que o câncer pode gerar no indivíduo, ressalta-se que a prática da espiritualidade promove melhor aceitação do câncer e do tratamento, além de níveis mais elevados de esperança e otimismo, gerando influência positiva na dimensão biopsicossocial e espiritual do paciente oncológico. Isso reflete em melhora no bem-estar, na qualidade de vida, no controle do estresse, na diminuição da dor e melhora da disposição ${ }^{11}$.

Sendo assim, a presente revisão integrativa da literatura se faz relevante devido a todas as adversidades que enfrentam o paciente oncológico, que vão desde o diagnóstico, perpassando por todo período de tratamento, podendo o exercício da espiritualidade ser um auxílio para o enfrentamento desse processo. O conhecimento da importância da dimensão espiritual contribuirá para o estímulo a mais estudos e sensibilização por parte dos profissionais de saúde para desenvolvimento do cuidado espiritual no paciente oncológico. Para isso, este estudo busca responder a seguinte questão norteadora: para pacientes com câncer, o exercício da espiritualidade contribui para o tratamento oncológico? E tem como objetivo: analisar as evidências científicas sobre o exercício da espiritualidade em pacientes com câncer, tendo em vista suas contribuições para o tratamento oncológico.

\section{Metodologia}

Trata-se de uma revisão integrativa da literatura. Este tipo de estudo contempla resultados relevantes obtidos por diferentes autores acerca de uma mesma temática, de forma a agregar conceitos e informações para a construção do conhecimento científico baseado 
em evidências ${ }^{12}$.

O desenvolvimento desta revisão integrativa ocorreu nas seguintes etapas: 1 - Escolha da temática a ser buscada. 2 - Elaboração da questão norteadora da pesquisa. 3 - Escolha das bases de dados. 4 - Escolha dos descritores e elaboração das estratégias de busca. 5 - Elaboração de critérios de inclusão e exclusão. 6 - Busca na base de dados e 7 - Análise dos dados encontrados ${ }^{13}$.

Frente ao tema em estudo, a elaboração da pergunta de pesquisa foi norteada utilizando-se a estratégia PICO, que representa um acrônimo onde $\mathrm{P}=$ população, $\mathrm{I}=$ intervenção, $\mathrm{C}=$ comparação e $\mathrm{O}=$ desfecho ou resultado. Esse método facilita a captação de termos objetivos para o direcionamento da busca na literatura ${ }^{14}$. Visto que esta revisão não tem como foco pesquisas clínicas, o item $\mathrm{C}$ foi desconsiderado para a elaboração da pergunta do estudo, sendo estruturado da seguinte forma: $\mathrm{P}=$ pacientes em tratamento oncológico; $\mathrm{I}=$ exercício espiritual; $\mathrm{C}=$ não se aplica; e $\mathrm{O}=$ existência de contribuições para o tratamento oncológico.

As bases de dados utilizadas para a busca foram a Medical Literature Analysis and Retrieval System Online (MEDLINE), Literatura Latina Americana e do Caribe em Ciências em Saúde (LILACS), Banco de Dados em Enfermagem (BDENF) e base de dados cinzenta (google scholar e referências bibliográficas). As buscas foram realizadas no mês de junho de 2020 utilizando os seguintes Descritores em Ciências da Saúde (DECS): "Espiritualidade", "Oncologia" e "Adaptação psicológica", termos estes que mais se aproximaram do objeto do estudo. O operador booleano "AND" foi utilizado nas diferentes combinações dos DECS durante as buscas.

Foram adotados os seguintes critérios de inclusão: artigos originais, publicados entre os anos de 2010 a 2020, nos idiomas português, inglês e espanhol, e que abordassem a temática proposta. Foram excluídas: teses; dissertações; materiais não disponíveis na íntegra de forma gratuita e artigos duplicados. A partir da utilização da estratégia de busca, foram encontrados 864 artigos científicos, destes, 849 foram excluídos, sendo 32 por estarem duplicados, 671 por não atenderam à questão norteadora; 7 por estarem sem resumo; 71 por não ser originais; 36 por estarem indisponíveis; e 32 por estarem publicados em outra língua. Sendo incluídos para análise da revisão 15 artigos. Conforme pode ser visualizado no fluxograma de seleção dos artigos, baseado no modelo PRISMA (figura 1).

Após a seleção, os 15 artigos foram organizados de acordo com o ano de publicação, o periódico de publicação, os títulos, os nomes dos (as) autores (as), os objetivos e classificação de nível de evidência (NE), sendo os seguintes níveis analisados de acordo com a hierarquização na qual se encaixa: I - revisões

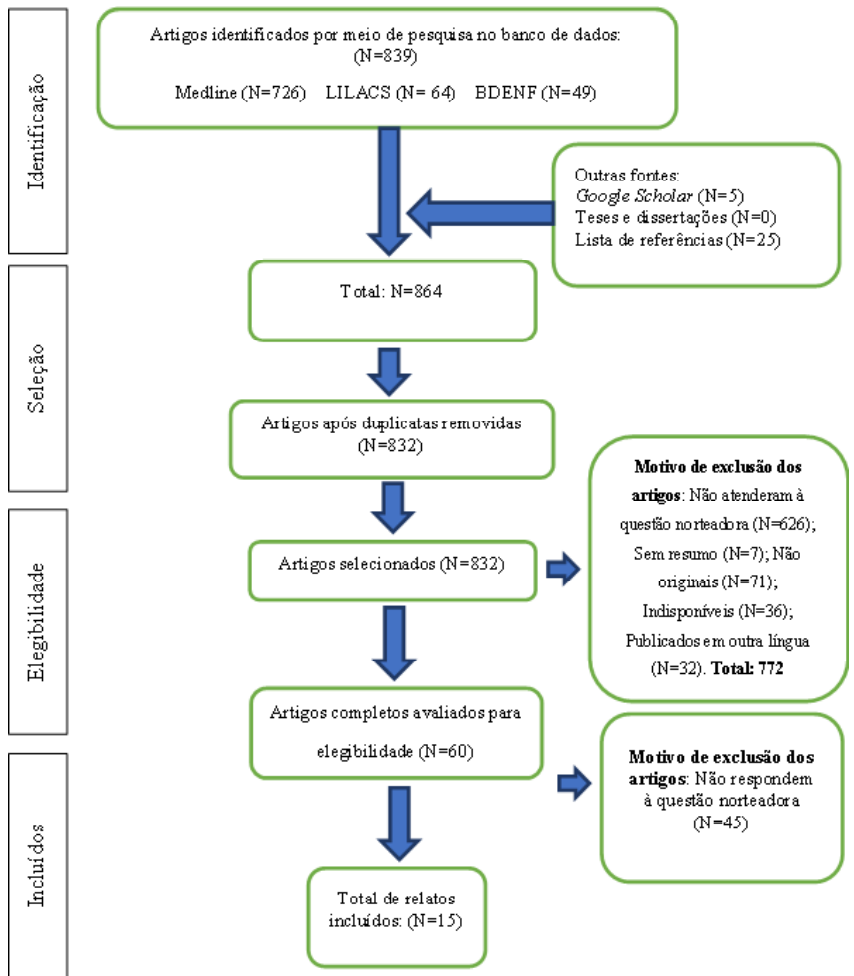

Figura 1. Fluxograma de seleção dos artigos para a revisão de literatura acerca da contribuição da espiritualidade para pacientes com câncer em tratamento oncológico.

sistemáticas ou metanálise de relevantes ensaios clínicos; II - evidências de, pelo menos, um ensaio clínico randomizado controlado bem delineado; III ensaios clínicos bem delineados sem randomização; IV estudos de coorte e de caso-controle bem delineados; V revisão sistemática de estudos descritivos e qualitativos; VI - evidências derivadas de um único estudo descritivo ou qualitativo; VII - opinião de autoridades ou comitês de especialistas incluindo interpretações de informações não baseadas em pesquisas ${ }^{13}$.

A análise dos dados resultou na categorização dos resultados encontrados nos artigos selecionados para a revisão integrativa: Categoria 1: A espiritualidade e o bem-estar físico e emocional; Categoria 2: A espiritualidade na construção da resiliência; e Categoria 3: A espiritualidade ressignificando a vida.

Esta pesquisa foi realizada conforme as determinações éticas da Lei de Direitos Autorais, Lei ${ }^{\circ}$ 12.853, de 14 de agosto de 2013, respeitando os direitos autorais dos artigos coletados. $O$ presente estudo não necessitou de aprovação em comitê de ética por ser uma revisão integrativa da literatura ${ }^{15}$.

\section{Resultados}

Foram selecionados quinze artigos que abordaram o papel da espiritualidade durante o tratamento oncológico. Destes, $40 \%(\mathrm{n}=6)$ foram obtidos na base 
de dados MEDLINE, 6,6\% $(\mathrm{n}=1)$ na base de dados LILACS e $53,3 \%(\mathrm{n}=8)$ na literatura cinzenta. Com relação ao periódico, $40 \%(\mathrm{n}=6)$ foram publicados em periódicos estrangeiros e $60 \%(\mathrm{n}=9)$ em nacionais. De acordo com ano de publicação, $20 \%(\mathrm{n}=3)$ no ano de $2011,20 \%(n=3) 2015,13,3 \%(n=2) 2010$ e $6,6 \%(n=1)$ para cada ano de 2012, 2013, 2017, 2018, 2019 e 2020.

Quanto aos países onde os estudos foram realizados, a maioria, $60 \%(\mathrm{n}=9)$, foi no Brasil e os outros 6 artigos da amostra cada um foi distribuído nas seguintes localidades: China, em três países da União Europeia (Portugal, Espanha e Suíça), nos Estados Unidos da América, na França, na Itália e na Malásia. Em relação ao idioma de publicação, $60 \%(\mathrm{n}=9)$ em português e $40 \%(\mathrm{n}=6)$ em inglês. Quanto ao método do artigo, $60 \%(\mathrm{n}=9)$ qualitativo, $33,3 \%(\mathrm{n}=5)$ quantitativo e $6,6 \%(n=1)$ qualitativo e quantitativo. Com relação ao nível de evidência, todos os artigos utilizados para análise estão classificados no nível VI (evidências derivadas de um único estudo descritivo ou qualitativo). O quadro 1 apresenta a relação de artigos selecionados de acordo com o ano, periódico, título do artigo, autores e objetivo.

Notou-se que, apesar da espiritualidade e a religiosidade não serem sinônimos, todos os artigos nacionais $^{16-24}$ e alguns artigos internacionais ${ }^{25,26}$ possuem participantes que relacionam o exercício espiritual diretamente à prática religiosa, enquanto os demais estudos levam em conta outras atividades, como meditação, atividades diárias, literatura, arte, musicoterapia e relacionamentos interpessoais ${ }^{27,28,30}$.

Os estudos selecionados abordaram diferentes papéis atribuídos à prática espiritual, a saber: a espiritualidade como apoio para o diagnóstico de câncer e as dificuldades do tratamento, fonte de ânimo para enfrentar as adversidades e ressignificação da vida e da própria existência, desta forma, estabeleceu-se três categorias para melhor apresentação e discussão dos resultados. Categoria 1: A espiritualidade e o bem-estar físico e emocional; Categoria 2: A espiritualidade na construção da resiliência; e Categoria 3: A espiritualidade ressignificando a vida.

\section{Discussão}

\section{A espiritualidade e o bem-estar fisico e emocional}

Alguns artigos analisados trazem como resultados que o tratamento para o câncer pode gerar baixa autoestima, autocrítica e dificuldade de aceitação e de enfrentamento da mudança de vida, podendo afetar os comportamentos e desencadear depressão. E que o exercício da espiritualidade promove o bem-estar físico e emocional para esses pacientes ${ }^{16,24,26,27,29}$.

A dificuldade de aceitação da doença e seu tratamento podem levar à depressão acompanhada de sentimento de culpa, raiva, apatia e tristeza, com desdobramentos negativos para $\mathrm{o}$ enfrentamento do câncer, o que pode acarretar na negligência do autocuidado $^{17}$. Porém, esse quadro é capaz de ser revertido com a prática espiritual, que leva o indivíduo a ter maior aceitação e disposição para o tratamento oncológico proposto e a desenvolver uma atitude positiva para consigo mesmo, melhorando o humor e a disposição para atividades de lazer ${ }^{27,29}$.

A espiritualidade está relacionada a fatores que afetam aspectos sociais, familiares, emocionais e funcionais ${ }^{28}$. A qualidade de vida, por sua vez, apesar de ser um parâmetro subjetivo, está diretamente relacionada ao bem-estar espiritual e pessoal, bem como com a autoestima $^{31}$. Assim, atividades como relaxamento, meditação, oração e religiosidade são práticas que refletem de forma eficaz no bem-estar e na qualidade de vida dos indivíduos em tratamento oncológico ${ }^{28}$.

Nesse ponto, percebe-se que o exercício da espiritualidade, independente da sua forma, funciona como proteção a agravos psicoemocionais, facilita a aceitação do diagnóstico de câncer e contribui para o bem estar e qualidade de vida do indivíduo, por reforçar a fé, a esperança e facilitar o uso de estratégias de enfrentamentos.

Sabe-se, contudo, que um dos fatores mais determinantes do sofrimento físico e emocional durante o tratamento do câncer é a dor ${ }^{32}$. A percepção da dor oncológica ocorre de forma singular e sofre a influência de fatores diversos, como o medo, o sentimento de revolta, a raiva e o estresse, perpassando pelas dimensões física, espiritual, emocional e social ${ }^{6}$. Para tanto, se faz relevante conhecer os efeitos da espiritualidade neste quesito. Observou-se que as práticas espirituais funcionam como fator protetor contra a dor e demais sintomas oncológicos, aumentando assim a qualidade de vida dos pacientes durante o tratamento ${ }^{30}$. Nesse sentido, aqueles pacientes que recorrem à oração como método para sentir alívio físico e mental, apresentam maior estabilidade emocional e bem-estar geral ${ }^{16,17,20,22,}$ 30

Ademais, é possível perceber que os artigos trazem a oração como uma atividade espiritual que gera alívio e paz. Sendo esta uma prática que promove a conexão a um ser superior a quem podem confiar e desabafar angustias e apreensões, em um processo que favorece a redução da sobrecarga emocional, física e espiritual impostas ao paciente.

Conviver com o câncer é pesaroso, porém quando o indivíduo se concentra em atividades espirituais, a forma de ver o contexto em que está inserido sofre mudanças positivas, capazes de colaborar para a adoção de hábitos de vida mais saudáveis ${ }^{21,26}$. Essa nova postura adotada por indivíduos que exercitam a espiritualidade produz otimismo, bem-estar, qualidade 
Quadro 1. Artigos selecionados de acordo com o ano, periódico, título, autores e objetivos..

\begin{tabular}{|c|c|c|c|c|}
\hline Ano & Periódico & Título & Autores & Objetivos \\
\hline 2010 & $\begin{array}{l}\text { Psicologia: } \\
\text { Teoria e } \\
\text { Pesquisa }\end{array}$ & $\begin{array}{c}\text { Religiosidade/ } \\
\text { Espiritualidade Em } \\
\text { Pacientes } \\
\text { Oncológicos: } \\
\text { Qualidade De Vida E } \\
\text { Saúde }\end{array}$ & $\begin{array}{l}\text { Fornazari SA, } \\
\text { Ferreira } \\
\text { RER }^{16}\end{array}$ & $\begin{array}{c}\text { Investigar o } \\
\text { enfrentamento religioso } \\
\text { em pacientes oncológicos }\end{array}$ \\
\hline 2010 & $\begin{array}{c}\text { Palliative and } \\
\text { Supportive } \\
\text { Care }\end{array}$ & $\begin{array}{l}\text { Do spirituality and faith } \\
\text { make a difference? } \\
\text { Report from the Southern } \\
\text { European Psycho- } \\
\text { Oncology Study Group. }\end{array}$ & $\begin{array}{c}\text { Travado L, } \\
\text { Grassi L, Gil } \\
\text { F, et } \mathrm{al}^{29}\end{array}$ & $\begin{array}{c}\text { Examinar o efeito da } \\
\text { espiritualidade em moldar } \\
\text { as implicações } \\
\text { psicossociais em pacientes } \\
\text { com câncer do sul da } \\
\text { Europa. } \\
\end{array}$ \\
\hline 2011 & J Relig Health & $\begin{array}{l}\text { Religion and spirituality } \\
\text { in coping with advanced } \\
\text { breast cancer: } \\
\text { perspectives from } \\
\text { Malaysian Muslim } \\
\text { women. }\end{array}$ & $\begin{array}{c}\text { Ahmad F, } \\
\text { Muhammad } \\
\text { MB, Abdulla } \\
\text { AA }^{26}\end{array}$ & $\begin{array}{l}\text { Desvelar o significado da } \\
\text { experiência de } \\
\text { espiritualidade ao } \\
\text { enfrentarem os desafios do } \\
\text { câncer de mama. }\end{array}$ \\
\hline 2011 & $\begin{array}{l}\text { Revista } \\
\text { Brasileira de } \\
\text { Enfermagem }\end{array}$ & $\begin{array}{l}\text { Relação entre } \\
\text { espiritualidade e câncer: } \\
\text { perspectiva do paciente }\end{array}$ & $\begin{array}{l}\text { Guerrero GP, } \\
\text { Zago MMFI, } \\
\text { Sawada NO, } \\
\text { et } \mathrm{al}^{18}\end{array}$ & $\begin{array}{l}\text { Compreender a relação } \\
\text { entre espiritualidade e o } \\
\text { câncer na perspectiva de } \\
\text { pacientes oncológicos }\end{array}$ \\
\hline 2011 & $\begin{array}{l}\text { Psycho- } \\
\text { Oncology }\end{array}$ & $\begin{array}{l}\text { Evaluation of } \\
\text { intrapsychic factors, } \\
\text { coping styles, and } \\
\text { spirituality of patients } \\
\text { affected by tumors }\end{array}$ & $\begin{array}{l}\text { Vespa A, } \\
\text { Jacobsen PB, } \\
\text { Spazzafumo } \\
\text { L, et } \mathrm{al}^{27}\end{array}$ & $\begin{array}{c}\text { Estabelecer se uma } \\
\text { perspectiva espiritual está } \\
\text { correlacionada com } \\
\text { habilidades de } \\
\text { enfrentamento e processos } \\
\text { intrapsíquicos mais } \\
\text { eficazes. }\end{array}$ \\
\hline 2012 & $\begin{array}{c}\text { Revista } \\
\text { Interdisciplina } \\
\text { r Saúde e } \\
\text { Meio } \\
\text { Ambiente }\end{array}$ & $\begin{array}{c}\text { A Influência Da } \\
\text { Religiosidade/Espirit } \\
\text { ualidade Na } \\
\text { Qualidade De Vida } \\
\text { Das Pessoas Com } \\
\text { Câncer }\end{array}$ & $\begin{array}{c}\text { Geronasso } \\
\mathrm{MCH}, \text { Coelho } \\
\mathrm{D}^{21} .\end{array}$ & $\begin{array}{l}\text { Lançar um olhar para as } \\
\text { influências da } \\
\text { religiosidade/ } \\
\text { espiritualidade na } \\
\text { qualidade de vida das } \\
\text { pessoas acometidas de } \\
\text { câncer. } \\
\end{array}$ \\
\hline 2013 & $\begin{array}{l}\text { Psychology, } \\
\text { Health \& } \\
\text { Medicine }\end{array}$ & $\begin{array}{l}\text { Two years after cancer } \\
\text { diagnosis, what is the } \\
\text { relationship between } \\
\text { health-related quality of } \\
\text { life, coping strategies } \\
\text { and spirituality? }\end{array}$ & $\begin{array}{c}\text { Préau M, } \\
\text { Bouhnik AD, } \\
\text { Soriano } \\
\text { AGLC }^{25}\end{array}$ & $\begin{array}{l}\text { Analisar a relação entre } \\
\text { espiritualidade, estratégias } \\
\text { de enfrentamento e } \\
\text { qualidade de vida entre } \\
\text { pacientes com dois anos } \\
\text { de diagnóstico de câncer. }\end{array}$ \\
\hline 2015 & $\begin{array}{l}\text { Psicologia } \\
\text { Ciência e } \\
\text { Profissão }\end{array}$ & $\begin{array}{c}\text { Espiritualidade, } \\
\text { Depressão e Qualidade } \\
\text { de Vida no } \\
\text { Enfrentamento do } \\
\text { Câncer: Estudo } \\
\text { Exploratório }\end{array}$ & $\begin{array}{l}\text { Miranda SL, } \\
\text { Lanna MAL, } \\
\text { Felippe } \mathrm{WC}^{24} \text {. }\end{array}$ & $\begin{array}{c}\text { Investigar inter-relações } \\
\text { entre bem estar espiritual, } \\
\text { depressão e qualidade de } \\
\text { vida durante o } \\
\text { enfrentamento do câncer }\end{array}$ \\
\hline
\end{tabular}


de vida, adaptação psicológica diante das adversidades e comportamentos vantajosos e construtivos ${ }^{33,34}$.

Estudos recentes trazem ainda que a prática espiritual pode afetar positivamente as funções imunológicas do indivíduo com câncer, isso porque o cortisol, que se eleva facilmente com estresse psicossocial, suprime o sistema imunológico. Por tanto, o estímulo ao bem-estar psicológico por meio da espiritualidade ajuda a manter níveis adequados de cortisol, contribuindo de forma eficaz para o bem-estar físico do paciente em tratamento oncológico ${ }^{35}$.

A religiosidade é uma ferramenta para o exercício espiritual, alguns artigos referem que por meio da prática religiosa é possível promover, além da melhoria da qualidade de vida, o controle das inseguranças relacionadas à doença e a redução da ansiedade ${ }^{16,36}$. Essa contribuição é importante porque a ansiedade, apesar de ser uma emoção natural do ser humano, em excesso pode gerar prejuízos significativos na vida do indivíduo ${ }^{36}$

O aumento de aspectos positivos, relacionados à prática espiritual, como o otimismo, esperança, adoção de hábitos de vida saudáveis e a redução do estresse e da ansiedade, esteve presente nos resultados de grande parte dos artigos utilizados na análise. Assim, ressaltase a importância do exercício da espiritualidade na vida dos pacientes com câncer, com desdobramentos que refletem no bem-estar físico e emocional.

Contrapondo os resultados das pesquisas apresentadas, um estudo realizado na França observou que $54,1 \%$ de uma amostra de 4.270 participantes com câncer, embora buscasse conforto na espiritualidade, concluiu que ela não traz benefícios concretos. Refere ainda que quanto maior o interesse por atividades espirituais, menor a qualidade de vida mental e física ${ }^{25}$. É possível que estes resultados tenham sido obtidos devido à associação da espiritualidade à religiosidade tendenciada pelos próprios autores que questionaram os participantes quanto à importância da religião, e não da espiritualidade, durante o enfrentamento oncológico. Este estudo não traz dados com relação à prática religiosa dos participantes.

Tal resultado foi também encontrado em estudo realizado no Brasil com 10 participantes, onde observou que alguns participantes não vêm a prática espiritual como importante, não atribuindo a ela função benéfica para o tratamento. Esses achados foram associados à singularidade de cada indivíduo, que encontrará ou não benefícios na prática espiritual de acordo com suas experiências, cultura e valores pessoais ${ }^{23}$.

Desta forma, é preciso compreender quais são as práticas espirituais que estão relacionadas a um maior benefício para o paciente oncológico, e em que ela pode contribuir para diminuição dos danos físicos e emocionais ocasionados pelo diagnóstico de câncer e seu tratamento.

\section{A espiritualidade na construção da resiliência}

A resiliência é definida como um processo de diferentes trajetórias caracterizado por bem-estar e capacidade de recuperar-se psicologicamente em circunstâncias que demandam esforços de enfrentamento por parte do indivíduo, como no caso das adversidades que surgem com o tratamento oncológico. Ser resiliente, por tanto, pode potencializar e multiplicar os resultados positivos do enfrentamento e minimizar o sofrimento ${ }^{37}$.

O exercício espiritual pode ser visto como uma ferramenta para construção da resiliência, por ter um importante impacto na forma como a pessoa enfrenta o câncer e o seu tratamento ${ }^{4}$. No percurso do adoecimento surgem dificuldades que geram sentimentos de medo e de impotência, e a espiritualidade colabora para atenuação desses aspectos e auxilia na busca pela saúde. Assim, pacientes que exercem práticas espirituais possuem maior otimismo em relação ao prognóstico, o que resulta em atitudes mais ativas e renovação da esperança na recuperação $0^{29}$.

Os pacientes oncológicos que apresentam maior adesão às práticas que estimulam a espiritualidade possuem melhores mecanismo de enfrentamento de situações estressantes, pois têm melhor atitude consigo mesmos, como autocuidado e autorrealização, maior facilidade de desenvolver suas qualidades pessoais, maior espontaneidade e capacidade de reduzir e controlar a angústia. Dessa forma, se adaptam melhor ao tratamento do câncer por estarem mais resilientes ${ }^{27}$, 24

Estes resultados ressaltam a capacidade que os pacientes, que exercem alguma prática espiritual, possuem de desenvolver a resiliência no enfrentamento do câncer e do seu tratamento. Ser resiliente envolve resistência, força, saber superar situações adversas e recuperar-se, por tanto, essa valiosa característica oferece à pessoa adoecida uma gama de mecanismos que o apoiam durante o tratamento oncológico ${ }^{4}$.

A resiliência auxilia no enfrentamento do medo e do desconhecido, os artigos estudados referem que o medo, apesar de ser natural ao ser humano, quando em excesso, pode trazer prejuízos ao paciente oncológico e a espiritualidade ajuda na superação ou atenuação deste sentimento. Um estudo realizado na Malásia trouxe que, ao se voltarem para a espiritualidade, mulheres com câncer de mama, perceberam que seus medos diminuíram, passando a se sentir mais fortes para enfrentar o tratamento ${ }^{26}$.

O sentimento produzido pelo exercício espiritual fortalece a resiliência, porque aumenta a confiança de que tudo ficará bem, devido à fé que se cria em um ser divino que age em favor de quem recorre a ele. Desenvolve-se a percepção de que o indivíduo não possui controle sobre a sua condição, mas alguém superior que preza pelo bem o tem, assim, lidar com o câncer se torna 
uma experiência mais tranquila. Além disso, a adesão ao tratamento se fortalece, visto que, diante da ideia de ser ajudado pela divindade, o paciente se vê ciente da necessidade de fazer a sua parte, para que assim seus esforços possam ser recompensados ${ }^{16,17,19,23}$.

$\mathrm{O}$ apego à espiritualidade contribui com o ajustamento à doença, afinal, gera um modo de pensar construtivo, mudanças de atitude, controle emocional e conforto ${ }^{22}$. Se por um lado ao receber o diagnóstico o medo prevalece, a espiritualidade entra como um fator renovador sobre o modo de pensar e encarar o tratamento ${ }^{18}$. Assim, a espiritualidade pode ser considerada como um alicerce para o enfretamento do processo de doença/tratamento e construção da resiliência ${ }^{23}$.

Além disso, constatou-se que pacientes em tratamento oncológico que praticam a espiritualidade se sentem impulsionados a ajudar outras pessoas na mesma situação de adoecimento. Eles se adaptam melhor ao tratamento do câncer devido à força, alívio, conforto, esperança, calma, tranquilidade, resiliência e superação dos medos, a partir da nova forma de pensar advinda do exercício espiritual ${ }^{17,19-21,23}$.

Dessa forma, percebe-se que as características positivas adquiridas através de atividades que estimulam a espiritualidade proporcionam apoio ao paciente ao ponto de se sentirem motivados a ajudar outros da mesma forma que se sentem ajudados. Esse altruísmo gera, ainda, sentimento recompensador nesses indivíduos, contribuindo também para o enfrentamento da própria doença.

No entanto, um dos artigos selecionados traz que muitos dos entrevistados não encontraram benefícios no exercício espiritual que pudessem auxiliá-los no enfrentamento do câncer. Os autores referem que muitos associavam a espiritualidade somente à pratica religiosa, e, dentre estes, muitos perceberam que esta atitude de busca espiritual não trouxe benefícios durante o tratamento do câncer. Isso pode estar relacionado ao excesso de expectativas projetadas a um ser superior que não foram atendidas, o que causou as frustrações relatadas no estudo com relação à espiritualidade ${ }^{25}$.

\section{A espiritualidade ressignificando a vida e a morte}

O câncer, bem como outras doenças, pode contribuir para ressignificação da vida e renovação dos valores $^{21}$. A maioria dos pacientes com câncer busca um sentido para a existência, um significado para vida e um propósito, e se sentem apoiados pela espiritualidade ${ }^{29}$. Isso é importante para que não haja, durante o processo de tratamento, a morbidade psicológica, ou seja, a depressão associada à ansiedade que, comumente, ocorre após diagnósticos negativos ${ }^{38,39,40}$.

A satisfação pessoal, o contato com sentimentos e emoções individuais, a autoapreciação e o equilíbrio emocional são frutos do desenvolvimento da espiritualidade durante o tratamento oncológico ${ }^{27}$. Dessa forma, as adversidades se transformam em algo significativo para a vida e a percepção do mundo se modifica $^{19}$

A espiritualidade se mostra importante para trazer significado e propósitoà vida das pessoas ${ }^{23}$. Concordando com isso, um estudo com mulheres em tratamento oncológico, constatou que o câncer representa uma experiência de aprendizagem, um despertar para a vida. A doença fez com que elas constassem que possuíam pouco controle sobre as circunstâncias, assim, com o exercício da espiritualidade, encontraram um maior significado para a existência. Elas passaram a considerar aspectos que, antes do diagnóstico da doença, não valorizavam e ressignificaram a visão que possuíam sobre a morte, não a encarando como um fracasso ${ }^{26}$.

A maioria dos estudos aqui analisados trazem que seus participantes, após o diagnóstico de câncer, buscaram através da espiritualidade um novo propósito para a existência. Alguns aspectos do cotidiano ganharam um outro significado diante da possibilidade de uma doença ameaçadora à vida, e a pratica espiritual foi fundamental para este processo. $\mathrm{O}$ adoecimento e a morte receberam uma nova representação, por estarem no controle de um ser supremo.

Muitos pacientes vão em busca do exercício espiritual, como fé, meditação, lazer e contato com amigos e familiares, para obter significado e passam a encarar o adoecimento como um desafio imposto que tem o objetivo de estimular o crescimento espiritual e pessoal ${ }^{16}$. Trazem que o diagnóstico de câncer amedronta, porém a espiritualidade tem o poder de trazer o renovo sobre essas circunstâncias, ou seja, apesar do câncer desestruturar os pacientes, a espiritualidade tem o poder de movê-los para a transformação da existência, de forma a enxergar o real significado da vida ${ }^{18}$.

\section{Conclusão}

A espiritualidade é compreendida como uma dimensão individual que envolve a busca do sentido da vida, da paz e do bem-estar, podendo ser praticada, ou não, de acordo com a necessidade, compreensão e circunstâncias de cada pessoa. Apesar de estar muito associada à religiosidade, as atividades que estimulam a espiritualidade podem envolver rotinas diárias, arte, música, leitura, oração e meditação.

Os estudos selecionados para esta revisão integrativa da literatura possibilitaram a verificação do papel da espiritualidade durante o tratamento oncológico. As pesquisas mostram a importância que é atribuída ao exercício espiritual pelos pacientes. Percebeu-se que na maioria das vezes a espiritualidade possui efeitos positivos, no entanto, em alguns casos, 
a prática espiritual pode não ter nenhum efeito sobre o indivíduo e, em menor quantidade, o efeito pode ser negativo.

Constatou-se que a prática espiritual é relevante para o bem-estar, qualidade de vida, enfrentamento de adversidades e ressignificação da existência durante o tratamento oncológico, contribuindo para construção da resiliência. Pode-se destacar também que a espiritualidade funciona como protetor contra agravos psicoemocionais, facilita a adaptação ao diagnóstico de câncer e contribui para a diminuição da dor.

Os artigos selecionados sugerem que mais estudos sejam realizados com essa temática de forma a trazer à atenção dos profissionais de saúde sobre a importância do incentivo ao exercício da espiritualidade, bem como treinar, capacitar e atualizar estes profissionais acerca de como desenvolver o cuidado espiritual em pacientes oncológicos.

Conforme o levantamento bibliográfico realizado, reforça-se a necessidade de novas pesquisas neste campo e maior abordagem do tema em cursos de graduação e pós graduação na área da saúde a fim de que essa dimensão espiritual do sujeito possa ser atendida, especialmente, no contexto do adoecimento. Há ainda conceitos e definições limitadas sobre a espiritualidade que precisam ser mais esclarecidos para uma melhor compreensão do papel da espiritualidade durante o tratamento oncológico

\section{Referências}

1. Thiengo PCS, Gomes AMT, Magno Conceição das Mercês MC, et al. Espiritualidade e religiosidade no cuidado em saúde: revisão integrativa. Cogitare enferm. 24: e58692, 2019. [acesso 2020 out 10] 24: e58692. Disponível em: https://revistas.ufpr.br/cogitare/article/view/58692/pdf.

2. Mendonça AB, Pereira ER, Barreto BMF, et al. Aconselhamento e assistência espiritual a pacientes em quimioterapia: uma reflexão à luz da Teoria de Jean Watson. Escola Anna Nery 22(4) 2018. [acesso em 2020 ou 10] 22(4). Disponível em: https://www.scielo.br/pdf/ean/v22n4/pt 14148145-ean-22-04-e20180081.pdf.

3. Filho LSM, Khoury HTT. Uso do Coping Religioso/Espiritual diante das Toxicidades da Quimioterapia no Paciente Oncológico. Revista Brasileira de Cancerologia 2018; 64(1): 27-33. [acesso em 2020 out 12] 64(1): 2733. Disponível em: https://rbc.inca.gov.br/revista/index.php/revista/article/ view/112/53.

4. Soratto MT, Silva DM, Zugno PI, et al. Espiritualidade e resiliência em pacientes oncológicos. Revista Saúde e Pesquisa, v. 9, n. 1, p. 53-63, jan./ abr. 2016. [acesso em 2020 set 20] v. 9, n. 1, p. 53-63. Disponível em: https:// periodicos.unicesumar.edu.br/index.php/saudpesq/article/view/4284/2749.

5. Horie LM, Barrére APN, Castro MG, et al. Diretriz BRASPEN de terapia nutricional no paciente com câncer. BRASPEN J 2019; 34 (Supl 1):2-32. [acesso em 2020 set 23] 34 (Supl 1):2-32. Disponível em: https:// faculdadebarretos.com.br/wp-content/uploads/2019/06/Diretriz_onco-2019separata.pdf.

6. Pedroso JKN, Diefenbach GD, Ilha S, et al. Dor em oncologia: percepção do paciente e dos profissionais de enfermagem. Revista Cubana de Enfermería. 2017;33(4):748-761. [acesso em 2020 set 22] 33(4):748761. Disponível em: http://www.revenfermeria.sld.cu/index.php/enf/article/ view/1020/309.

7. Silva NM, Santos MA, Oliveira RAA, et al. Idosos em Tratamento Quimioterápico: Relação entre Nível de Estresse, Sintomas Depressivos e Esperança. Psic.: Teor. E Pesq. [Internet], Brasília, 2019, [acesso em 2020 set 23] v. 35, e35441. Disponível em: https://www.scielo.br/scielo. php?script=sci_arttext\&pid=S0102-37722019000100541.

8. Gusmão AL. Espiritualidade e enfrentamento do câncer de mama em mulheres em processo de quimioterapia. [Dissertação] [Internet] Universidade Federal de Juiz de Fora, 2018. [acesso em 25 de outubro de 2020]. Disponível em: https://repositorio.ufjf.br/jspui/bitstream/ufjf/6822/1/ amandalopesgusmao.pdf

9. Meneguin S, Matos TDS, Ferreira MLSM. Percepcao de pacientes oncologicos em cuidados paliativos sobre qualidade de vida. Rev Bras Enferm [Internet]. 2018; [acesso em $2020 \mathrm{dez} 18$ ] 71(4):2114-20. Disponível em: https://www.scielo.br/pdf/reben/v71n4/pt 0034-7167-reben-71-041998.pdf.

10. Oliveira JM, Reis JB, Silva RA. Busca por cuidado oncológico: percepção de pacientes e familiares. Rev enferm UFPE on line., Recife, 2018. [acesso em 2020 maio 23] 12(4):938-46, abr. Disponível em: https:// pesquisa.bvsalud.org/portal/resource/pt/biblio-970474.

11. Ferreira LF, Freire AP, Silveira ALC. A Influência da Espiritualidade e da Religiosidade na Aceitação da Doença e no Tratamento de Pacientes Oncológicos: Revisão Integrativa da Literatura. Revista Brasileira de Cancerologia [Internet], 2020; [acesso em 2020 set 24] 66(2): e-07422. Disponível em: https://rbc.inca.gov.br/revista/index.php/revista/article/ view/422.

12. Crossetti MGO. Revisão integrativa de pesquisa na enfermagem o rigor cientifico que lhe é exigido. Rev. Gaúcha Enferm. [Internet], 2012. [acesso em 2020 out 02]. Disponível em: https:/www.scielo.br/pdf/rgenf/ v33n2/01.pdf.

13. Mendes KDS, Silveira RCCP, Galvão CM. Use of the bibliographic reference manager in the selection of primary studies in integrative reviews. Texto contexto - enferm. [Internet], 2019. [acesso em 2020 dez 04]. Disponível em: https://www.scielo.br/scielo.php?script=sci_arttext\&pid=S010407072019000100602 .

14. Santos CMC, Pimenta CAM, Nobre MRC. A estratégia pico para a construção da pergunta de pesquisa e busca de evidências. Rev Latino-am Enfermagem [Internet], 2007 maio-junho; [acesso em 2020 ago 29] 15(3). Disponível em: https://www.scielo.br/pdf/rlae/v15n3/pt_v15n3a23.pdf.

15. Lei no 12.853 de 14 de agosto de 2013. Altera os arts.5o, 68, 97, 98, 99 e 100 , acrescenta arts. $98-A, 98-B, 98-C, 99-A, 99-B, 100-A, 100-B$ e $109-A$ e revoga o art. 94 da Lei no 9.610, de 19 de fevereiro de 1998, para dispor sobre a gestão coletiva de direitos autorais, e dá outras providências. Diário Oficial da União [Internet], 2013. [acesso em $2020 \mathrm{dez}$ 05] Disponível em: http://www.planalto.gov.br/CCIVil 03/ Ato2011-2014/2013/Lei/L12853. htm.

16. Fornazari SA, Ferreira RER. Religiosidade/Espiritualidade em Pacientes Oncológicos: Qualidade de Vida e Saúde. Psicologia: Teoria e pesquisaabr [Internet], 2010, [acesso em 2020 set 10] Vol. 26 n. 2, pp. 265-272. Disponível em: https://www.scielo.br/scielo.php?pid=S0102$37722010000200008 \&$ script $=$ sci_abstract\&tlng=pt.

17. Freitas RA, Menezes TMO, Santos LB, et al. Espiritualidade e religiosidade no vivido do sofrimento, culpa e morte da pessoa idosa com câncer. Rev Bras Enferm [Internet], 2020; [acesso em 2020 out 03] 73. Disponível em: https://www.scielo.br/scielo.php?script=sci arttext\&pid=S0034-71672020001500154\&lng=es\&nrm=i\&tlng=pt.

18. Guerrero GP, Zago MMFI, Sawada NO, et al. Relação entre espiritualidade e câncer: perspectiva do paciente. Rev Bras Enferm, Brasília [Ineternet], 2011 jan-fev; [acesso em 2020 out 02] 64(1): 53-9. Disponível em: $\quad$ https://www.scielo.br/scielo.php?script=sci arttext\&pid=S003471672011000100008 .

19. Pallini AC, Ottati F, Cremasco GS, et al. Percepções de pacientes oncológicos sobre espiritualidade: Um estudo qualitativo. Revista Psicologia para America Latina [Internet], 2019. [acesso em 2020 out 05] n. 32, p. 169-179. Disponível em: http://pepsic.bvsalud.org/scielo.php?script=sci abstract\&pid=S1870-350X2019000200008

20. Souza VM, Frizzo HCF, Paiva MHP, et al. Espiritualidade, religiosidade e crenças pessoais de adolescentes com câncer. Rev Bras Enferm [Internet], 2015. [acesso em 2020 out 03] 68(5):791-6. Disponível em: https://www.scielo.br/pdf/reben/v68n5/0034-7167-reben-68-05-0791. pdf. 
21. Geronasso $\mathrm{MCH}$, Coelho D. A influência da religiosidade/ espiritualidade na qualidade de vida das pessoas com câncer. Saúde Meio Ambient [Internet], 2012. [acesso em 2020 out 02] V. 1, n. 1. Disponível em: http://www.periodicos.unc.br/index.php/sma/article/view/227.

22. Freire MEM, Vasconcelos MF, Silva TN, et al. Assistência espiritual e religiosa a pacientes com câncer no contexto hospitalar. J. Res.: fundam. Care. Online [Internet], 2017. [acesso em 2020 out 02] 9(2): 356-362. Disponível em: http://seer.unirio.br/index.php/cuidadofundamental/article/ view/4906/0.

23. Pinto AC, Marchesini SM, Zugno PI, et al. A importância da espiritualidade em pacientes com câncer. Rev.Saúde.Com [Internet], 2015; [acesso em 2020 out 02] 11(2): 114-122. Disponível em: https://online.unisc. br/seer/index.php/ripsunisc/article/view/13195.

24. Miranda SL, Lanna MAL, Felippe WC. Espiritualidade, Depressão e Qualidade de Vida no Enfrentamento do Câncer: Estudo Exploratório. Psicologia Ciência e Profissão [Internet], 2015. [acesso em 2020 out 03] vol. 35, núm. 3, pp. 870-885. Disponível em: https://www.scielo.br/scielo. php?pid=S1414-98932015000300870\&script=sci_abstract\&tlng=pt.

25. Préau M, Bouhnik AD, Soriano AGLC. Two years after cancer diagnosis, what is the relationship between healthrelated quality of life, coping strategies and spirituality? Psychology, Health \& Medicine [Internet], 2013. [acesso em 2020 mai 27] Vol. 18, No. 4, 375-386. Disponível em: https://pubmed.ncbi.nlm.nih.gov/23140373/.

26. Ahmad F, Muhammad MB, Abdulla, AA. Religion and Spirituality in Coping with Advanced Breast Cancer: Perspectives from Malaysian Muslim Women. J Relig Health [Internet], 2011; [acesso em 2020 julho 17] 50:3645. Disponível em: https://pubmed.ncbi.nlm.nih.gov/20924683/.

27. Vespa A, Jacobsen PB, Spazzafumo L, et al. Evaluation of intrapsychic factors, coping styles, and spirituality of patients affected by tumors. PsychoOncology [Inetrnet], 2011. [acesso em 2020 mai 24] 20: 5-11. Disponível em: https://pubmed.ncbi.nlm.nih.gov/20336646/.

28. Cheng Q, Liu X, Li X et al. Improving spiritual well-being among cancer patients: implications for clinical care. Supportive Care in Cancer [Internet], 2019. [acesso 2020 mai 22]. Disponível em: https://link.springer. com/article/10.1007/s00520-019-4636-4

29. Travado L, Grassi L, Gil F, et al. Do spirituality and faith make a difference? Report from the Southern European Psycho Oncology Study Group. Palliative and Supportive Care [Internet], 2010, [acesso em 2020 julho 10] 8, 405-413. Disponível em: https://www.cambridge.org/core/ journals/palliative-and-supportive-care/article/abs/do-spirituality-and-faithmake-a-difference-report-from-the-southern-european-psychooncologystudy-group/9EFD117AAEB7D2F84A522076205BE0C7.

30. Bai J, Brubaker A, Meghani SH et al. Spirituality and Quality of Life in Black Patients With Cancer Pain. Journal of pain and symptom management [Internet], 2018. [acesso em 27 de novembro de 2020]. Disponível em: https://www.jpsmjournal.com/article/S0885-3924(18)30287-2/fulltext.

31. Nunes MGS, Leal MCC, Marques APO, et al. Idosos longevos: avaliação da qualidade de vida no domínio da espiritualidade, da religiosidade e de crenças pessoais. Saúde Debate [Internet], 2017. [acesso em 2020 nov $28]$ v. 41, n. 115, p. 1102-1115. Disponível em: https://www.scielo.br/scielo. php?pid $=$ S0103-11042017000401102\&script $=$ sci_abstract\&tlng=pt.

32. Rangel O, Telles C. Tratamento da dor oncológica em cuidados paliativos. Rev HUPE [Internet], 2012. [acesso em 2020 nov 12] v. 11, n. 2, p. 32-7. Disponível em: https://www.e-publicacoes.uerj.br/index.php/ revistahupe/article/view/8928

33. Santos L, Faro A. Otimismo: Teoria e Aplicabilidade para a Psicologia. Revista Psicologia e Saúde [Internet], 2020. [acesso em 2020 nov 20] v. 12, n. 2, p. 123-139. Disponível em: http://pepsic.bvsalud.org/pdf/ rpsaude/v12n2/v12n2a09.pdf.

34. Santos MC, Wechsler SM. Escala de Otimismo para Adultos: Construcão e Validação. Psico-USF [Internet], 2020. [acesso em 2020 nov $20]$ v. 25 , n. 1, p. 89-100. Disponível em: https://www.scielo.br/pdf/pusf/ v25n1/2175-3563-pusf-25-01-89.pdf.

35. Seixas MC. Espiritualidade no contexto da saúde. Revista Unitas, [Internet], 2017. [acesso em 2020 dez 20] v.5, n.2 (n. especial). Disponível em: http://revista.fuv.edu.br/index.php/unitas/article/viewFile/652/541

36. Guimarães AMV, Neto ACS, Vilar ATS, et al. Transtornos de ansiedade: um estudo de prevalência sobre as fobias específicas e a importância da ajuda psicológica. Ciências Biológicas e da Saúde [Internet], 2015. [acesso em 2020 nov 23] v. 3, n.1, p. 115-128. Disponível em: https:// periodicos.set.edu.br/fitsbiosaude/article/view/2611/0

37. Brandão JM, Nascimento E. Resiliência psicológica: da primeira fase às abordagens baseadas em trajetória. Memorandum [Internet], 2019. [acesso em 2020 nov 26] 36. Disponível em: https://periodicos.ufmg.br/index.php/ memorandum/article/view/6875.

38. Mata LRF, de Carvalho EC, Gomes CRG, da Silva AC, Pereira MG. Autoeficácia e morbidade psicológica no pós-operatório de prostatectomia radical. Rev. Latino-Am. Enfermagem. [Internet] 2015;23(5) [acesso em 02 de dezembro de 2020]. Disponível: http://dx.doi.org/10.1590/01041169.0456 .2618

39. Almeida VM. Propósito de vida, morbilidade psicológica e qualidade de vida na dor crónica. [Dissertação] [Internet] Universidade do Minho; 2018. [acesso em 27 de novembro de 2020]. Disponível em: https://repositorium. sdum.uminho.pt/handle/1822/55939.

40. Moher D, Liberati A, Tetzlaff J, Altman DG, The PRISMA Group Preferred Reporting Items for Systematic Reviews and Meta-Analyses: The PRISMA Statement. Plos Med. [Internet], 2009; [acesso em 2020 set 01] 6(6):e1000097. Disponível em: https://pubmed.ncbi.nlm.nih. gov/19621072/. 